\title{
Efeito de herbicidas na dessecação e germinação de sementes remanescentes de Lolium multiflorum $\mathrm{L}$.
}

\section{Effect of herbicides on desiccation of Lolium multiflorum L. plants and seed germination}

\author{
Caio Ferraz de Campos ${ }^{1 *}$; Dagoberto Martins ${ }^{2}$; Andréia Cristina Peres Rodrigues da \\ Costa $^{3}$; Maria Renata Rocha Pereira ${ }^{4}$ Leonildo Alves Cardoso ${ }^{5}$. Cibele Chalita Martins ${ }^{6}$
}

\section{Resumo}

O objetivo desse trabalho foi o de avaliar a eficácia de diferentes herbicidas aplicados em pósemergência na dessecação de plantas de azevém e viabilidade de sementes remanescentes do controle, com diferentes volumes de calda de aplicação. Os tratamentos testados foram: três formulações de glyphosate na dose de $1.080 \mathrm{~g} \mathrm{ha}^{-1}$ (Roundup Original, Roundup Transorb, Roundup WG), paraquat + diuron nas doses de $300+150$ e $500+250 \mathrm{~g} \mathrm{ha}^{-1}$ e amônio-glufosinate nas doses de 400 e $600 \mathrm{~g}$ ha-

${ }^{1}$. Em todos os tratamentos foram utilizados os volumes de aplicação de $200 \mathrm{e} 400 \mathrm{~L} \mathrm{ha}^{-1}$. A eficácia na dessecação de plantas de azevém foram avaliados aos 1, 3, 7, 14 e 21 dias após aplicação, sendo que nesta última avaliação foram coletadas plantas para a análise de produção de matéria seca, assim como panículas com sementes para testar a sua viabilidade. A dessecação do azevém com o herbicida glyphosate nas diferentes formulações e volumes testados, bem como o herbicida amônio-glufosinate na dose de $600 \mathrm{~g} \mathrm{ha}^{-1}$ no volume de aplicação de $400 \mathrm{~L} \mathrm{ha}^{-1}$ proporcionaram os melhores controles. Os tratamentos com gliphosate (Roundup Original) e paraquat + diuron $\left(300+150 \mathrm{~g} \mathrm{ha}^{-1}\right)$, nos volumes de 400 e 200L há ${ }^{-1}$, respectivamente, foram os que proporcionaram os menores valores de matéria seca, diferindo da testemunha. Todas as sementes remanescentes de azevém foram mortas pelos tratamentos com os herbicidas paraquat + diuron na dose de $500+250 \mathrm{~g} \mathrm{ha}^{-1}$ e amônio-glufosinate na dose de $600 \mathrm{~g}$ $\mathrm{ha}^{-1}$, ambas com volume de aplicação de $200 \mathrm{~L} \mathrm{ha}^{-1}$.

Palavras-chave: Azevém, controle, tecnologia de aplicação, herbicida

\begin{abstract}
A trial was carried out to evaluate efficacy of different herbicides and spray volumes when sprayed to ryegrass for chemical management and control of remaining seeds. The treatments were: three formulations of glyphosate at $1080 \mathrm{~g} \mathrm{ha}^{-1}$ (Original Round up, Round up Transorb, WG Round Up), paraquat + diuron at $300+500$ and $150+250 \mathrm{~g} \mathrm{ha}^{-1}$ and glufosinate ammonium at 400 and $600 \mathrm{~g} \mathrm{ha}^{-1}$. All treatments were used in spray volumes of 200 and $4001 \mathrm{ha}^{-1}$. The effectiveness of the desiccation of ryegrass plants was assessed at 1, 3, 7, 14 and 21 days after application. At the last evaluation samples were collected for dry mass production analysis, and also panicles with seeds for germination test. Glyphosate in different formulations and volumes and glufosinate ammonium at $600 \mathrm{~g} \mathrm{ha}^{-1}$ when

${ }^{1}$ Discente de Pós-Graduação, Faculdade de Ciências Agronômicas,Universidade Estadual Paulista Júlio de Mesquita Filho, UNESP, Botucatu, SP. E-mail: caio.agro@hotmail.com

2 Prof. Adjunto, Dept ${ }^{\circ}$ de Produção Vegetal, FCA/UNESP, Botucatu, SP. E-mail: dmartins@fca.unesp.br

3 Bolsista Pós-Doutorado, Universidade Estadual do Oeste do Paraná, CCA/UNIOESTE, Cascavel, PR. E-mail: andreiacpr@,

${ }^{4}$ Prof ${ }^{a}$ da Faculdade de Tecnologia em Silvicultura, Campus Capão Bonito, SP. E-mail: mariarenata10@hotmail.com

${ }_{5}$ Dr. em Agronomia, Consultor Nacional da FAO, Food and Agriculture Organization, em Guiné-Bissau, África. E-mail: leonildocardoso@hotmail.com

${ }^{6}$ Prof $^{\mathrm{a}} \mathrm{Dr}^{\mathrm{a}}$, Dept ${ }^{\circ}$ de Produção Vegetal, FCAV/UNESP, Jaboticabal, SP. E-mail: cibele@fcav.unesp.br Autor para correspondência
\end{abstract} hotmail.com 
sprayed with into $4001 \mathrm{ha}^{-1}$ provided the best controls regarding ryegrass chemical management. Treatments with gliphosate (Roundup Original) and paraquat + diuron $\left(300+150 \mathrm{~g} \mathrm{ha}^{-1}\right)$ in volumes of 400 and $200 \mathrm{~L} \mathrm{ha}^{-1}$, respectively, were the ones that showed the lowest values of dry matter, differing from the control. All the ryegrass seeds were killed by treatments with herbicides (paraquat + diuron) at $500+250 \mathrm{~g} \mathrm{ha}^{-1}$ and glufosinate-ammonium at $600 \mathrm{~g} \mathrm{ha}^{-1}$ when used $2001 \mathrm{ha}^{-1}$.

Key words: Ryegrass, control, spraying technology, herbicide

\section{Introdução}

O azevém (Lolium multiflorum L.), de fecundação cruzada, é uma gramínea de inverno, com ciclo anual, utilizada principalmente como forrageira e para fornecimento de palha ao sistema plantio direto. É uma espécie que se adapta bem a solos de baixa e média fertilidade, com boa resposta à adubação, de fácil dispersão e, por isso, está presente e caracterizase como planta daninha em praticamente todas as lavouras de inverno, em pomares e em vinhedos da região Sul do Brasil. Essa gramínea também é considerada problema em lavouras de verão, como o milho (VARGAS; MORAES; BERTO, 2007). Assim, torna-se necessário realizar a operação de manejo com máxima eficácia, evitando que imprecisões na aplicação prejudiquem a instalação da cultura de verão (MATUO, 1990).

A dessecação das plantas daninhas ou de culturas utilizadas em rotação apresenta grande importância para o estabelecimento de uma lavoura, visto que a emergência destas, juntamente com a cultura, provocam danos tanto na produtividade como na qualidade dos grãos. O azevém tem-se apresentado como um importante problema na cultura do trigo, estando, muitas vezes, já presente por ocasião da semeadura da cultura. Os produtores têm encontrado normalmente dificuldade para controlar o azevém com o herbicida glyphosate, prática comumente realizada (ROMAN et al., 2004b).

O eficiente controle químico das plantas de cobertura torna-se fundamental para o sucesso no estabelecimento principalmente de culturas graníferas. Assim, o controle das plantas de cobertura e da infestação por plantas daninhas antes da semeadura permite que a cultura tenha um desenvolvimento inicial livre de interferências, o que impede rebrotas e reinfestações na área, facilitando a ação de herbicidas complementares, após a emergência da cultura (ALMEIDA, 1991).

Vários pesquisadores já relataram prejuízos causados em lavouras cultivadas com o convívio de plantas de azevém. Segundo Appleby, Olson e Colbert (1976) tem-se uma queda de 7 a 50\% na produtividade do trigo quando na presença de plantas de azevém. Já, Liebel e Worsham (1987) afirmam a existência de uma redução na produção de trigo na ordem de $4,2 \%$ a cada 10 plantas de azevém por metro quadrado. Também, Fleck (1980) observou um decréscimo linear com o aumento da população de azevém na cultura do trigo.

A eficiência de herbicidas no controle de plantas daninhas também depende do volume de água usada como veículo em sua aplicação. Tem-se observado que a eficiência do herbicida glyphosate aumenta quando se diminui o volume de calda de pulverização. Essa melhor eficiência tem sido atribuída à melhor cobertura da folhagem (AMBACH; ASHFORD, 1982) e à maior concentração de ingrediente ativo nas gotas da pulverização (JORDAN, 1981).

A baixa eficiência na dessecação de plantas é uma grande preocupação nos campos de cultivos, pois sementes remanescentes podem infestar os cultivos sucessivos. Dada a natureza e o modo de ação de produtos utilizados para dessecação, são necessárias pesquisas sobre possíveis danos latentes, os quais poderiam encurtar a longevidade da semente (DOMINGOS; SIVLA; SILVA, 2000).

Avaliando características fisiológicas, bioquímicas e sanitárias de sementes de soja colhidas após dessecação com quatro dessecantes, Lacerda et al. (2003) observou que a germinação de sementes provenientes de plantas dessecadas 
com glufosinato de amônio foi menor, quando comparada com a dessecação feita com paraquat, diquat e suas misturas.

Assim, o presente trabalho teve como objetivo avaliar a eficácia de diferentes herbicidas aplicados em pós-emergência na dessecação do azevém e na germinação das sementes remanescentes, com diferentes volumes de calda de aplicação.

\section{Material e Métodos}

O presente trabalho foi instalado e conduzido em condição de campo, na Fazenda Experimental Lageado da Faculdade de Ciências Agronômicas - FCA/UNESP, campus de Botucatu/SP. As coordenadas geográficas da área são: Latitude $22^{\circ} 07^{\prime} 56^{\prime \prime} \mathrm{S}$ e Longitude $74^{\circ}$ 66' 84" WGr., com altitude média de $762 \mathrm{~m}$ acima do nível do mar, precipitação média anual de $1.517 \mathrm{~mm}$ e a temperatura média anual de $20,6^{\circ} \mathrm{C}$. O clima da região é, pela classificação por Koppen, como Cfa, ou seja, subtropical, com verões quentes e úmidos e com invernos frios e secos.

O delineamento experimental foi de blocos casualizados, com quatro repetições. As parcelas experimentais foram constituídas de 8 linhas de semeadura com $4 \mathrm{~m}$ de comprimento, espaçadas de $0,25 \mathrm{~m}$ entre si, totalizando $8 \mathrm{~m}^{2}$. Foi considerada como área útil as 6 linhas centrais e também desconsiderou-se $0,5 \mathrm{~m}$ das extremidades de cada parcela. O preparo do solo foi realizado mecanicamente, através de uma aração com arado de aiveca, gradagem com uma grade pesada e duas operações de grade niveladora. A semeadura foi realizada no dia 20/03/2008. As sementes foram distribuídas manualmente no sulco de semeadura em quantidade necessária para alcançar 60 plantas de azevém por metro linear. Foram realizadas capinas manuais durante todo o período experimental, e duas adubações nitrogenadas (40Kg ha $\left.{ }^{-1} \mathrm{~N}\right) \mathrm{em}$ cobertura realizada na cultura aos 30 e 45 dias após a emergência.

Os tratamentos testados estão apresentados na Tabela 1. A aplicação dos tratamentos foi realizada 120 dias após a semeadura no dia 19/07/2008. Para a aplicação dos herbicidas foi utilizado um pulverizador costal, pressurizado a $\mathrm{CO}_{2}$, equipado com uma barra de pulverização munida com quatro pontas de jato plano tipo XR 11002 VS, com pressão de trabalho de $200 \mathrm{kPa}$, para um consumo de calda de 200L ha ${ }^{-1}$, e pontas XR 11004 VS com pressão de trabalho de $380 \mathrm{kPa}$, para um consumo de calda de 400L ha-1. As condições climáticas no momento da aplicação foram: temperatura (inicial de $19,0^{\circ} \mathrm{C}$ e final $18,0^{\circ} \mathrm{C}$ ), umidade relativa (inicial 51,0 e final $68,0 \%)$ e ventos na forma de rajadas $(2 \mathrm{~km} / \mathrm{h})$.

Os efeitos dos tratamentos na dessecação das plantas de azevém foram avaliados visualmente, por escala de percentual de notas, na qual 0 (zero) corresponde a nenhuma injúria demonstrada pela planta e 100 (cem) à morte das plantas, segundo a Sociedade Brasileira da Ciência das Plantas Daninhas - SBCPD (1995). As avaliações de controle das plantas de azevém foram realizadas aos 1, 3, 7, 14 e 21 dias após a aplicação (DAA) dos herbicidas. Aos 21 DAA foram coletadas as plantas presentes em duas das linhas centrais, sendo $1 \mathrm{~m}$ de cada linha, as quais foram secas em estufa de ventilação forçada de ar a $60 \pm 5^{\circ} \mathrm{C}$, até atingir peso constante e, em seguida, foi determinada a matéria seca das amostras. Os parâmetros utilizados para o estabelecimento das notas visuais de controle foram: inibição do crescimento, quantidade e uniformidade das injúrias, capacidade de rebrota das plantas e quantidade de plantas mortas. 
Tabela 1. Herbicidas, dose e volume de calda utilizados na dessecação da cultura do azevém (Lolium multiflorum). Botucatu/SP, 2008.

\begin{tabular}{llcc}
\hline \multicolumn{1}{c}{ Tratamento } & Produto Comercial & Volume de calda ( $\left.\mathbf{l} \mathbf{~ h a}^{-\mathbf{1}}\right)$ & Dose (g i.a/e.a. $\left.\mathbf{~ h a}^{-\mathbf{1}}\right)$ \\
\hline 1. glyphosate & Roundup Original & 200 & 1.080 \\
2. glyphosate & Roundup Original & 400 & 1.080 \\
3. glyphosate & Roundup Transorb & 200 & 1.080 \\
4. glyphosate & Roundup Transorb & 400 & 1.080 \\
5. glyphosate & Roundup WG & 200 & 1.080 \\
6. glyphosate & Roundup WG & 400 & 1.080 \\
7. (paraquat+diuron) & Gramocil & 200 & $300+150$ \\
8. (paraquat+diuron) & Gramocil & 400 & $300+150$ \\
9. (paraquat+diuron) & Gramocil & 200 & $500+250$ \\
10. (paraquat+diuron) & Gramocil & 400 & $500+250$ \\
11. amônio-glufosinate & Finale & 200 & 400 \\
12. amônio-glufosinate & Finale & 400 & 400 \\
13. amônio-glufosinate & Finale & 200 & 600 \\
14. amônio-glufosinate & Finale & 400 & 600 \\
15. testemunha & -- & -- & -- \\
\hline
\end{tabular}

Fonte: Elaboração dos autores.

Foram colhidas 20 panículas de forma aleatória de cada parcela para realização do teste de germinação. Foi utilizada uma solução de $0,2 \%$ de $\mathrm{KNO}_{3}$ para superação de dormência das sementes. No tese de germinação utilizou-se 100 sementes de cada parcela que foram semeadas sobre duas folhas de papel germitest umedecidas com 2,5 vezes seu peso com a solução de $\mathrm{KNO}_{3}$, em caixas plásticas transparentes, tipo gerbox, no qual se utilizou o delineamento experimental inteiramente casualizado, com quatro repetições. As avaliações foram executadas de acordo com as Regras para Análise de Sementes (BRASIL, 2009), computandose as porcentagens de plântulas normais para as repetições nos períodos de tempo recomendados. As caixas foram acondicionadas individualmente em sacos plásticos de $0,05 \mathrm{~mm}$ de espessura para a manutenção da umidade do substrato (GASPAR et al., 2007), e em seguida acondicionadas em germinadores sob regime alternado de temperatura e de luz $\left(30{ }^{\circ} \mathrm{C}\right.$ por 8 horas e $20{ }^{\circ} \mathrm{C}$ por 16 horas) (BRASIL, 2009).

Os resultados foram submetidos a analise de variância pelo teste "F" a $5 \%$ de probabilidade, sendo que os valores de porcentagem de germinação foram transformados segundo arco seno $\sqrt{\mathrm{x}} / 100$, e as medias comparadas pelo teste de Tukey $(p>0,05)$.

\section{Resultados e Discussão}

Observou-se, já no primeiro dia após a aplicação (DAA) dos herbicidas, que as plantas de azevém apresentaram sintomas de fitointoxicação em todos os tratamentos testados (Tabela 2), destacando-se os tratamentos com paraquat + diuron na dose mais elevada $\left(500+250 \mathrm{~g} \mathrm{ha}^{-1}\right)$, independente do volume de aplicação e o tratamento com os herbicidas (paraquat + diuron) na dose de $300+150 \mathrm{~g} \mathrm{ha}^{-1}$ aplicado no volume de $200 \mathrm{~L} \mathrm{ha}^{-1}$ proporcionaram níveis de injúrias em torno de 11,0 a 12,25\%.

Verifica-se, aos 3 DAA, que todos os tratamentos proporcionaram incrementos em seu controle, com comportamento semelhante ao observado ao 1 DAA, com destaque aos tratamentos com os herbicidas paraquat + diuron na dose de $500+250$ $\mathrm{g} \mathrm{ha}^{-1}$ em ambos os volumes de aplicação testados, com fitotoxicidade acima de $50 \%$, e o tratamento com paraquat + diuron na dose de $300+150 \mathrm{~g} \mathrm{ha}^{-1}$ 
no volume de $200 \mathrm{~L} \mathrm{ha}^{-1}$ (Tabela 2). Essa rápida ação da mistura paraquat + diuron deveu-se ao efeito de contato proporcionado pelo paraquat, o qual necessita de apenas 1 a 3 dias para provocar necrose completa das partes vegetais atingidas (AHRENS, 1994).

Na avaliação realizada aos 7 DAA os tratamentos com o herbicida glyphosate, independente da formulação e do volume de aplicação, foram os que apresentaram os piores resultados no controle do azevém, todos os demais tratamentos proporcionaram controles que variaram de 44,25 a $63,25 \%$ (Tabela 2). Tal fato ocorreu devido à ação lenta do herbicida glyphosate, pois este herbicida tem ação sistêmica e demanda um período maior de tempo para demonstrar sua eficiência. Quando se analisa os resultados observados aos 14 DAA, com exceção dos herbicidas paraquat + diuron na dose de $300+150 \mathrm{~g} \mathrm{ha}^{-1}$, todos os tratamentos testados proporcionaram um controle acima de $71 \%$; ressalta-se que todos os tratamentos com o herbicida glyphosate proporcionaram os melhores resultados com controle acima de $80 \%$.

Tabela 2. Porcentagem de fitointoxicação visual em plantas de Lolium multiflorum sobre o efeito de herbicidas aplicados em pós-emergência. Botucatu/SP, 2008.

\begin{tabular}{|c|c|c|c|c|c|c|c|}
\hline \multirow{2}{*}{ Tratamentos } & \multirow{2}{*}{$\begin{array}{c}\text { Dose } \\
\text { (g i.a/e.a. } \\
\left.\text { ha }^{-1}\right)\end{array}$} & \multirow{2}{*}{$\begin{array}{l}\text { Volume } \\
\left(\mathrm{L} \mathrm{ha}^{-1}\right)\end{array}$} & \multicolumn{5}{|c|}{ Dias Após a Aplicação (DAA) } \\
\hline & & & 1 & 3 & 7 & 14 & 21 \\
\hline 1.glyphosate ${ }^{11}$ & 1.080 & 200 & $1,00 \mathrm{c}$ & $3,00 \mathrm{~cd}$ & $25,50 \mathrm{~d}$ & $88,75 \mathrm{a}$ & $80,50 \mathrm{ab}$ \\
\hline 2.glyphosate ${ }^{11}$ & 1.080 & 400 & $0,50 \mathrm{c}$ & $1,75 \mathrm{~cd}$ & $21,50 \mathrm{~d}$ & $80,50 \mathrm{ab}$ & $82,00 \mathrm{ab}$ \\
\hline 3.glyphosate ${ }^{12}$ & 1.080 & 200 & $1,25 \mathrm{c}$ & $2,50 \mathrm{~cd}$ & $24,00 \mathrm{~d}$ & $80,50 \mathrm{ab}$ & $80,50 \mathrm{ab}$ \\
\hline 4.glyphosate ${ }^{12}$ & 1.080 & 400 & $1,00 \mathrm{c}$ & $1,75 \mathrm{~cd}$ & $22,00 \mathrm{~d}$ & $82,50 \mathrm{ab}$ & $81,25 \mathrm{ab}$ \\
\hline 5.glyphosate ${ }^{3}$ & 1.080 & 200 & $0,75 \mathrm{c}$ & $2,00 \mathrm{~cd}$ & $32,75 \mathrm{~cd}$ & $84,75 \mathrm{ab}$ & $87,75 \mathrm{a}$ \\
\hline 6.glyphosate ${ }^{13}$ & 1.080 & 400 & $0,50 \mathrm{c}$ & $2,00 \mathrm{~cd}$ & $25,00 \mathrm{~d}$ & $83,25 \mathrm{ab}$ & $87,00 \mathrm{a}$ \\
\hline 7. (paraquat+diuron) & $300+150$ & 200 & $12,25 \mathrm{a}$ & $46,75 \mathrm{ab}$ & $45,00 \mathrm{abc}$ & $65,75 \mathrm{bc}$ & $51,75 \mathrm{c}$ \\
\hline 8. (paraquat+diuron) & $300+150$ & 400 & $6,25 \mathrm{~b}$ & $37,75 \mathrm{~b}$ & $44,25 \mathrm{bc}$ & $53,25 \mathrm{c}$ & $46,75 \mathrm{c}$ \\
\hline 9. (paraquat+diuron) & $500+250$ & 200 & $11,25 \mathrm{a}$ & $53,25 \mathrm{a}$ & $55,00 \mathrm{ab}$ & $77,75 \mathrm{ab}$ & $65,00 \mathrm{bc}$ \\
\hline 10. (paraquat+diuron) & $500+250$ & 400 & $12,00 \mathrm{a}$ & $56,75 \mathrm{a}$ & $63,25 \mathrm{a}$ & $80,75 \mathrm{ab}$ & $63,25 \mathrm{bc}$ \\
\hline 11.amônio-glufosinate & 400 & 200 & $1,50 \mathrm{c}$ & $6,75 \mathrm{~cd}$ & $44,00 \mathrm{bc}$ & $71,25 a b c$ & $61,75 \mathrm{bc}$ \\
\hline 12.amônio-glufosinate & 400 & 400 & $2,25 \mathrm{c}$ & $11,75 \mathrm{c}$ & $53,00 \mathrm{ab}$ & $76,75 \mathrm{ab}$ & $76,25 \mathrm{ab}$ \\
\hline 13.amônio-glufosinate & 600 & 200 & $1,75 \mathrm{c}$ & $9,00 \mathrm{~cd}$ & $46,25 \mathrm{abc}$ & $76,75 \mathrm{ab}$ & $67,00 \mathrm{abc}$ \\
\hline 14.amônio-glufosinate & 600 & 400 & $1,50 \mathrm{c}$ & $6,75 \mathrm{~cd}$ & $54,25 \mathrm{ab}$ & $88,25 \mathrm{a}$ & $82,00 \mathrm{ab}$ \\
\hline 15.testemunha & -- & -- & $0,00 \mathrm{c}$ & $0,00 \mathrm{~d}$ & $0,00 \mathrm{e}$ & $0,00 \mathrm{~d}$ & $0,00 \mathrm{~d}$ \\
\hline $\mathrm{F}_{\text {tratamento }}$ & & & $41,61^{* *}$ & $96,78^{* *}$ & $23,15^{* *}$ & $34,34^{* *}$ & $29,33^{* *}$ \\
\hline C.V. $(\%)$ & & & 39,0 & 26,3 & 19,2 & 10,4 & 12,3 \\
\hline $\mathrm{dms}$ & & & 3,56 & 10,81 & 18,14 & 19,16 & 21,18 \\
\hline
\end{tabular}

** significativo a $1 \%$.

Médias seguidas de mesma letra na coluna não diferem estatisticamente entre si pelo teste " $\mathrm{t}$ " ( $\mathrm{p}>0,05)$.

$\backslash 1$ Roundup Original \2Roundup Transborb \3 Roundup WG.

Fonte: Elaboração dos autores.

No final do estudo, aos 21 DAA, verifica-se que os únicos herbicidas que não proporcionaram rebrotas das plantas de azevém foram o Roundup Original quando aplicado em 400L ha-1 e o Roundup WG independente do volume de aplicação testado.
Os tratamentos que apresentaram os menores valores no controle das plantas de azevém, foram os que continham o herbicida paraquat + diuron, independente da dose e volume, e os tratamentos com o herbicida amônio-gluphosinate nas duas 
doses testadas, ambas no volume de $2001 \mathrm{ha}^{-1}$. A reduzida translocação dos herbicidas paraquat + diuron inviabiliza sua utilização isolada em áreas que apresentam infestação de gramíneas perenes, fato que pode ser revertido se posicionado de forma seqüencial, após a aplicação de glyphosate (PROCÓPIO et al., 2006). Esses dados coincidem com os encontrados por Marochi (1995), que verificou alta porcentagem de rebrota de gramíneas com a utilização de paraquat + diuron isoladamente ou em aplicação sequencial, recomendando a utilização de herbicida sistêmico na primeira aplicação. Ressalta-se que quando aplicado o herbicida amônio-glufosinate, independente das doses testadas, porém a 400L ha-1 foi observado maiores níveis de intoxicação das plantas em relação a $200 \mathrm{~L} \mathrm{ha}^{-1}$, esse resultado contradiz o encontrado por Roman et al. (2004a), para uma outra espécie que observaram redução de $10 \%$ no controle de Brachiaria plantaginea, quando se aumentou o volume de aplicação.

Ao analisar-se o acúmulo de massa seca registrase que todos os tratamentos apresentaram valores inferiores de massa seca em relação testemunha (Tabela 3). Os tratamentos apresentaram comportamento semelhante, com valores de massa seca acima de $3.000 \mathrm{Kg} \mathrm{ha-1}$, com exceção aos tratamentos com os herbicidas glyphosate (Roundup Original) aplicado no volume de calda de $400 \mathrm{~L} \mathrm{ha}^{-1}$ e paraquat + diuron na dose de $300+150 \mathrm{~g} \mathrm{ha}^{-1}$ no volume de $200 \mathrm{~L} \mathrm{ha}^{-1}$ que apresentou redução da massa seca em 38,12 e $42,79 \%$, respectivamente, em relação a testemunha.

Tabela 3. Efeito de diferentes herbicidas aos 21 dias após a pulverização em pós-emergência sobre a produção de massa seca em plantas de Lolium muliflorum. Botucatu/SP, 2008.

\begin{tabular}{|c|c|c|c|}
\hline Tratamento & $\begin{array}{c}\text { Dose } \\
\left.\text { (g i.a/e.a. } \text { ha }^{-1}\right)\end{array}$ & $\begin{array}{c}\text { Volume de calda } \\
\quad\left(\mathbf{l ~ h a}^{-1}\right)\end{array}$ & $\begin{array}{c}\text { M. S. } \\
\left(\mathrm{Kg} \mathrm{ha}^{-1}\right)\end{array}$ \\
\hline 1. glyphosate ${ }^{11}$ & 1.080 & 200 & $3.634,50 \mathrm{ab}$ \\
\hline 2. glyphosate $e^{11}$ & 1.080 & 400 & $2.835,00 \mathrm{~b}$ \\
\hline 3. glyphosate ${ }^{12}$ & 1.080 & 200 & $3.137,75 \mathrm{ab}$ \\
\hline 4. glyphosate ${ }^{12}$ & 1.080 & 400 & $3.587,00 \mathrm{ab}$ \\
\hline 5. glyphosate ${ }^{13}$ & 1.080 & 200 & $3.263,00 \mathrm{ab}$ \\
\hline 6. glyphosate ${ }^{13}$ & 1.080 & 400 & $3.846,25 \mathrm{ab}$ \\
\hline 7. (paraquat+diuron) & $300+150$ & 200 & $2.620,75 \mathrm{~b}$ \\
\hline 8. (paraquat + diuron) & $300+150$ & 400 & $3.569,00 \mathrm{ab}$ \\
\hline 9. (paraquat+diuron) & $500+250$ & 200 & $3.966,00 \mathrm{ab}$ \\
\hline 10. (paraquat+diuron) & $500+250$ & 400 & $3.491,00 \mathrm{ab}$ \\
\hline 11. amônio-glufosinate & 400 & 200 & $3.692,75 \mathrm{ab}$ \\
\hline 12. amônio-glufosinate & 400 & 400 & $3.766,75 \mathrm{ab}$ \\
\hline 13. amônio-glufosinate & 600 & 200 & $3.513,00 \mathrm{ab}$ \\
\hline 14. amônio-glufosinate & 600 & 400 & $3.948,00 \mathrm{ab}$ \\
\hline 15. testemunha & -- & -- & $4.581,25 \mathrm{a}$ \\
\hline $\mathrm{F}_{\text {tratamento }}$ & & & $1,98^{*}$ \\
\hline $\mathrm{CV}(\%)$ & & & 19,0 \\
\hline $\mathrm{dms}$ & & & $1.723,86$ \\
\hline
\end{tabular}

*significativo a $5 \%$.

Médias seguidas de mesma letra na coluna não diferem estatisticamente entre si pelo teste " $t$ " ( $p>0,05)$.

$\backslash 1$ Roundup Original \2Roundup Transborb \3 Roundup WG

Fonte: Elaboração dos autores. 
Embora não tenha ocorrido diferença estatística em alguns tratamentos, a germinação das sementes foi afetada pelos diferentes herbicidas, apresentando reduções na germinação das sementes de azevém comparando-se com a testemunha (Tabela 4), bem como o vigor das sementes, medida pelo teste da $1^{\mathrm{a}}$. contagem. $\mathrm{O}$ efeito mais drástico sobre a germinação ocorreu com os tratamentos utilizandose os herbicidas paraquat+diuron na dose de 500
$+250 \mathrm{~g} \mathrm{ha}^{-1}$ e amônio-glufosinate na dose de $600 \mathrm{~g}$ $\mathrm{ha}^{-1}$, ambos com volume de aplicação de $200 \mathrm{~L}$ $\mathrm{ha}^{-1}$, apresentaram controle total na germinação das sementes coletadas, assim evitando uma futura infestação na cultura subsequente. Tal fato demonstra que nem sempre os tratamentos com os melhores controles visuais das plantas são os que reduzem mais a germinação das sementes das plantas remanescentes na área tratada.

Tabela 4. Efeito de diferentes herbicidas sobre a germinação de sementes de Lolium multiflorum, submetidos à ação de diferentes herbicidas e volumes e calda de pulverização. Botucatu/SP, 2008.

\begin{tabular}{|c|c|c|c|c|}
\hline \multirow{2}{*}{ Tratamento } & \multirow{2}{*}{$\begin{array}{c}\text { Dose } \\
\left.\text { (g i.a/e.a. ha' }{ }^{-1}\right)\end{array}$} & \multirow{2}{*}{$\begin{array}{c}\text { Volume de calda } \\
\quad\left(\mathrm{L} \mathrm{ha}^{-1}\right)\end{array}$} & \multicolumn{2}{|c|}{ Teste de Germinação } \\
\hline & & & $1^{\mathrm{a}}$ contagem $(\%)$ & Normais (\%) \\
\hline 1. glyphosate & 1.080 & 200 & $3,00 \mathrm{ab}$ & $6,75 \mathrm{ab}$ \\
\hline 2. glyphosate ${ }^{11}$ & 1.080 & 400 & $0,75 \mathrm{ab}$ & $2,75 \mathrm{bc}$ \\
\hline 3. glyphosate ${ }^{12}$ & 1.080 & 200 & $1,00 \mathrm{ab}$ & $3,75 \mathrm{abc}$ \\
\hline 4. glyphosate ${ }^{12}$ & 1.080 & 400 & $1,25 \mathrm{ab}$ & $2,75 \mathrm{bc}$ \\
\hline 5. glyphosate ${ }^{13}$ & 1.080 & 200 & $0,75 \mathrm{ab}$ & $4,00 \mathrm{bc}$ \\
\hline 6. glyphosate ${ }^{13}$ & 1.080 & 400 & $1,00 \mathrm{ab}$ & $6,75 \mathrm{ab}$ \\
\hline 7. (paraquat+diuron) & $300+150$ & 200 & $0,25 \mathrm{ab}$ & $1,00 \mathrm{bc}$ \\
\hline 8. (paraquat+diuron) & $300+150$ & 400 & $1,00 \mathrm{ab}$ & $5,25 \mathrm{abc}$ \\
\hline 9. (paraquat+diuron) & $500+250$ & 200 & $0,00 \mathrm{~b}$ & $0,00 \mathrm{c}$ \\
\hline 10. (paraquat+diuron) & $500+250$ & 400 & $0,75 \mathrm{ab}$ & $1,25 \mathrm{bc}$ \\
\hline 11. amônio-glufosinate & 400 & 200 & $0,25 \mathrm{ab}$ & $0,50 \mathrm{bc}$ \\
\hline 12. amônio-glufosinate & 400 & 400 & $0,00 \mathrm{~b}$ & $5,50 \mathrm{ab}$ \\
\hline 13. amônio-glufosinate & 600 & 200 & $0,00 \mathrm{~b}$ & $0,00 \mathrm{c}$ \\
\hline 14. amônio-glufosinate & 600 & 400 & $1,75 \mathrm{ab}$ & $6,00 \mathrm{ab}$ \\
\hline 15. testemunha & -- & - & $3,75 \mathrm{a}$ & $15,25 \mathrm{a}$ \\
\hline $\mathrm{F}_{\text {tratamento }}$ & & & $2,78^{*}$ & $5,28^{* *}$ \\
\hline $\mathrm{CV}(\%)$ & & & 104,2 & 54,7 \\
\hline $\mathrm{dms}$ & & & 9,75 & 12,83 \\
\hline
\end{tabular}

* Significativo a 5\%, ** significativo a $1 \%$.

Médias seguidas de mesma letra na coluna não diferem estatisticamente entre si pelo teste " $\mathrm{t}$ " ( $\mathrm{p}>0,05)$.

Comparação feita com transformação em arco seno $\sqrt{x} / 100$.

$\backslash 1$ Roundup Original $\backslash 2$ Roundup Transborb $\backslash 3$ Roundup WG

Fonte: Elaboração dos autores.

\section{Referências}

AHRENS, W. H. Herbicide handbook. 7. ed. Champaign: Weed Science Society of America, 1994. 352 p.

ALMEIDA, F. S. Controle de plantas daninhas em plantio direto. Londrina: IAPAR, 1991. 34 p. (Circular, $67)$.

AMBACH, R. M.; ASHFORD, R. Effects of variation in drop makeup on the phytotoxicity of glyphosate. Weed
Science, Champaign, v. 30, n. 3, p. 221-224, 1982.

APPLEBY, A. P.; OLSON, P. D.; COLBERT, D. R. Winter wheat yield reduction from interference by Italian ryegrass. Agronomy Journal, Madison, v. 68, n. 3, p. 463466, 1976.

BRASIL. Ministério da Agricultura, Pecuária e Abastecimento. Regras para análise de sementes. Brasília: MAPA/ACS, 2009. 399 p. 
DOMINGOS, M.; SILVA, A. A.; SILVA, J. F. Qualidade da semente de feijão armazenada após dessecação química das plantas, em quatro estádios de aplicação. Acta Scientiarum. Maringá, v. 22, n. 4, p. 1143-1148, 2000.

FLECK, N. G. Competição de azevém (Lolium multiflorum L.) com duas cultivares de trigo. Planta Daninha, Viçosa, MG, v. 3, n. 2, p. 61-67, 1980.

GASPAR, C. M.; MARTINS, C. C.; NAKAGAWA, J.; TOMAZ, C. A. Manutenção da umidade do substrato durante o teste de germinação de Brachiaria brizantha. Revista Brasileira de Sementes, Londrina, v. 29, n. 3, p. 52-60, 2007.

JORDAN, T. N. Effects of diluent volumes and surfactant on the phytotoxicity of glyphosate to bemudagrass (Cynodon dactilon). Weed Science, Champaign, v. 29, n. 1, p. 79-83, 1981.

LACERDA, A. L. S.; LAZARINI, E.; SÁ, M. E.; VALÉRIO FILHO, W. V. Armazenamento de sementes de soja dessecadas e avaliação da qualidade fisiológica, bioquímica e sanitária. Revista Brasileira de Sementes, Brasília, v. 25, n. 2, p. 97-105, 2003.

LIEBEL, R.; WORSHAM, A. D. Effect of chlorsulfuron on diclofop phytotoxicity to Italian ryegrass (Lolium multiflorum). Weed Science, Champaign, v. 35, n. 3, p. 383-387, 1987.

MAROCHI, A. I. Evaluaton of chemical control methods for Richardia brasiliensis in infested areas under no-till system in the southern region of Brazil. In: ZAPP THE CHALLENGE OF THE NEW, 1995, São Paulo. Abstracts... São Paulo: [s.n], 1995. p. 57-60.

MATUO, T. Técnicas de aplicação de defensivos agrícolas. Jaboticabal: FUNEP, 1990. 139 p.

PROCÓPIO, S. O.; PIRES, F. R.; MENEZES, C. C. E.; BARROSO, A. L. L.; MORAES, R. V.; SILVA, M. V. V.; QUEIROZ, R. G.; CARMO, M. L. Efeitos de dessecantes no controle de plantas daninhas na cultura da soja. Planta Daninha, Viçosa, MG, v. 24, n. 1, p. 193-197, 2006.

ROMAN, E. S.; VARGAS, L.; RIBEIRO, M. C. F.; LUIZ, A. R. M. Influência do orvalho e volume de calda de aplicação na eficácia do glyphosate na dessecação de Brachiaria plantaginea. Planta Daninha, Viçosa, MG, v. 22, n. 3, p. 479-482, 2004a.

ROMAN, E. S.; VARGAS, L.; RIZZARDI, M. A.; MATTEI, R. W. Resistência de azevém (Lolium multiflorum) ao herbicida glyphosate. Planta Daninha, Viçosa, MG, v. 22, n. 2, p. 301-306, 2004b.

SOCIEDADE BRASILEIRA DA CIÊNCIA DAS PLANTAS DANINHAS - SBCPD. Procedimentos para instalação, avaliação e análise de experimentos com herbicidas. Londrina: SBCPD, 1995. 42 p.

VARGAS, L.; MORAES, R. M. A.; BERTO, C. M. Herança da resistência de azevém (Lolium multiflorum) ao glyphosate. Planta Daninha, Viçosa, MG, v. 25, n. 3, p. 567-571, 2007. 